\title{
Distinct gene-expression profiles characterize mammary tumors developed in transgenic mice expressing constitutively active and C-terminally truncated variants of STAT5
}

\section{Tali Eilon and Itamar Barash*}

\author{
Address: Institute of Animal Science, ARO, The Volcani Center, Bet-Dagan, Israel \\ Email: Tali Eilon - eilon@agri.huji.ac.il; Itamar Barash* - Barashi@agri.huji.ac.il \\ * Corresponding author
}

Published: I8 May 2009

BMC Genomics 2009, 10:231 doi:10.1|86/|47|-2164-10-231
Received: 4 December 2008

Accepted: 18 May 2009

This article is available from: http://www.biomedcentral.com//47/-2/64//0/23 I

(C) 2009 Eilon and Barash; licensee BioMed Central Ltd.

This is an Open Access article distributed under the terms of the Creative Commons Attribution License (http://creativecommons.org/licenses/by/2.0), which permits unrestricted use, distribution, and reproduction in any medium, provided the original work is properly cited.

\begin{abstract}
Background: Stat5 is a latent transcription factor that regulates essential growth and survival functions in normal cells. Constitutive activity of Stat5 and the involvement of its C-terminally truncated variant have been implicated in blood cell malignancies and mammary or breast cancer. To distinguish the individual contributions of the Stat5 variants to mammary tumorigenesis, global gene-expression profiling was performed on transgenic STAT5-induced tumors.
\end{abstract}

Results: We identified 364 genes exhibiting differential expression in mammary tumors developed in transgenic mice expressing constitutively active STAT5 (STAT5 ca) vs. its C-terminally truncated variant (STAT5 $\Delta 750)$. These genes mediate established Stat5 effects on cellular processes such as proliferation and cell death, as well as yet-unrelated homeostatic features, e.g. carbohydrate metabolism. A set of 14 genes linked STAT5 $\triangle 750$ expression to the poorly differentiated carcinoma phenotype and STAT5ca to the highly differentiated papillary adenocarcinoma.

Specifically affected genes exhibited differential expression in an individual tumor set vs. its counterpart and the intact mammary gland: 50 genes were specifically affected by STAT5ca, and $94 \%$ of these were downregulated, the latter involved in suppression of tumor suppressors and proliferation antagonistics. This substantial downregulation distinguishes the STAT5ca-induced tumorigenic consequences from the relatively equal effect of the STAT5 $\triangle 750$ on gene expression, which included significant elevation in the expression of oncogenes and growth mediators.

STAT5 5750 mRNA expression was below detection levels in the tumors and the amount of STAT5ca transcript was not correlated with the expression of its specifically affected genes. Interestingly, we identified several groups of three to eight genes affected by a particular STAT5 variant with significant correlated expression at distinct locations in the clustergram.

Conclusion: The different gene-expression profiles in mammary tumors caused by the STAT5 $\triangle 750$ and STAT5ca variants, corroborated by the absence of a direct link to transgenic STAT5 expression, imply distinct metabolic consequences for their oncogenic role which probably initiate early in tumor development. Tumorigenesis may involve induction of growth factor and oncogenes by STAT5 $\Delta 750$ or suppression of tumor suppressors and growth antagonists by STAT5 ca. The list of genes specifically affected by the STAT5 variants may provide a basis for the development of a marker set for their distinct oncogenic role. 


\section{Background}

Stat5 is a latent transcription factor that is activated upon binding of specific cytokines to their cognate membranal receptors. Activation of Stat 5 involves phosphorylation of specific tyrosine residues by Janus kinase 2 (Jak2), and the translocation of dimerized Stat5 molecules into the nucleus to bind target sites (TTCC $(\mathrm{A}>\mathrm{T}) \mathrm{GGAA}$ ) in individual gene promoters. Consequently, induction of a wide variety of signaling events ensues, observed mainly in the hematopoietic system and mammary gland. Stat5's transactivation domain (TAD) has been mapped to amino acids 750 to 772 in the C-terminal domain of the molecule [1]. This domain interacts with co-factors and is essential for transcription activation [2]. The TAD encompasses the main diversity between STAT5a, which is prevalent in the mammary gland, and Stat $5 b$ which is more abundant in the liver. Several naturally occurring C-terminally truncated variants of Stat5 have been identified. These variants are generated by alternative splicing or proteolytic cleavage, most commonly by nuclear serine proteases [3-5].

In the blood, naive T cells in the peripheral blood mononuclear cell fraction exclusively express the C-terminally truncated Stat5 [6]. Upon activation by mitogenic stimuli, the truncated Stat5a and Stat5b are replaced by the fulllength Stat5, implying that the truncated proteins have distinct functions. Naturally truncated forms of Stat 5 have been implicated in blood-cell cancer: 94\% of patients with relapsed leukemia expressed this Stat 5 variant, suggesting that it controls progression of the disease [7]. Constitutive activation of Stat5 has also been linked to a variety of blood-derived malignancies such as BCR-ABLinduced chronic myeloid leukemia (CML [8]), acute myeloid leukemia (AML) and acute lymphoid leukemia (ALL [9]).

In the mammary gland, Stat5's expression and activity are induced during pregnancy and lactation and decrease upon involution. During these stages, Stat 5 controls epithelial cell proliferation, final differentiation, lactogenesis, cell survival and tissue remodeling. These cellular processes play a major role in the structural and functional adaptation of the gland to the specific stages of the female reproductive cycle (reviewed in [10]). Overexpression of STAT5 in transgenic mice induced proliferation of mammary epithelial cells during pregnancy, increased $\beta$ casein synthesis upon lactation, and delayed involution [11]. In contrast, the C-terminally truncated variant was unable to induce $\beta$-casein/luciferase activity upon prolactin stimulation in vitro. Its expression in the mammary glands of transgenic animals resulted in reduced rates of cell proliferation at pregnancy and increased apoptosis during involution. Morphological signs of milk secretion upon parturition were delayed [12].
Stat 5 has also been associated with breast cancer. Stat5a nuclear localization was observed in $76 \%$ of breast cancer specimens and a positive correlation was established between its nuclear localization and the level of histological differentiation of the tumors [13]. Inactivation of Stat5a in transgenic mice expressing transforming growth factor (TGF) $\alpha$ or the SV40 T antigen delayed hyperplasia [14] and mammary cancer progression. More recently, a direct effect for Stat5 on mammary tumorigenesis was established $[15,16]$. Overexpression and forced activation of STAT5 caused parity-dependent development, most frequently of differentiated tumors, in transgenic post-estropausal female mice. Surprisingly, comparable rates of tumors $(\sim 8 \%)$ with similar latency periods were monitored in mice expressing the C-terminally truncated STAT5 protein, though the amount of poorly differentiated tumors in these mice was higher.

Both the constitutively active and C-terminally truncated variants of Stat 5 are potent oncogenes. In this study, we sought to determine the distinctness of their effects and the exclusive contribution of each variant to the oncogenic profiles of gene expression.

Profiling global gene expression in breast cancer has improved our understanding of the clinical diversity of this disease, allowing a better classification of its subtypes and definition of their response to drug treatment. Attempts to predict survival rates have also been reported [17-20]. Transgenic mouse models have been used to study the signatures of specific genes involved in initiation and maintenance of the disease. Genetic analysis of these mouse models could be highly relevant to human cancer [21-23]. The data also suggest that genes involved in the same pathway generate tumors with similar expression profiles, which are distinct from the profiles of tumors arising from other transgenic pathways [24].

Gene-expression profiles were compared in tumors caused by the constitutively active STAT5 (STAT5ca) and its C-terminally truncated variant (STAT5 4750$)$. A set of 364 differentially expressed genes was identified. Analysis and classification of these genes suggest that defined differences, possibly triggered at early stages of tumor development, characterize these Stat 5 variants.

\section{Methods}

\section{Mouse mammary-tumor samples}

Mammary tumors were derived from transgenic mice carrying one of two Stat 5 variants on a FVB/N background: (i) constitutively activated STAT5, termed STAT5ca, comprising sequences from three genes: amino acids 1-750 from ovine Stat5, which is homologous to mouse Stat5a, 677-847 from human Stat6, and 757-1129 from mouse Jak2 and (ii) a deleted construct, STAT $\Delta \Delta 750$, prepared by 
introducing a stop codon at the respective site of the native Stat5 DNA sequence, thus eliminating the expression of its TAD. These constructs were inserted into the $\beta$ lactoglobulin (BLG) multiple-cloning site for mammarygland-specific expression [11]. Upon identification, tumors were excised and snap-frozen for RNA isolation and validation studies. The pathological analysis of the tumors was performed by Dr. Robert Cardiff (University of California, Davis) as previously described [15]. All animals used in this study were treated humanely. Study protocols were in compliance with the regulations of the Israeli Ministry of Health and local institutional policies (approval no. IL- 39-03).

\section{RNA extraction and microarray hybridization}

The protocols for RNA extraction from tumors and mammary glands and microarray hybridization were as previously described [23]. Briefly, RNA was extracted from individual tumors or mammary glands with TRIZOL and reverse-transcribed. Equal amounts of complementary (c) RNA from each tumor were hybridized to an Affymetrix GeneChip ${ }^{\varpi}$ Mouse Genome 430A 2.0 array (Affymetrix, Santa Clara, CA), which includes approximately 14,000 annotated genes from the mouse genome. Hybridization and signal quantitation were performed according to Affymetrix's protocol by the Biological Services of the Weizmann Institute of Science (Rehovot, Israel). Total RNA $(15 \mu \mathrm{g})$ was reversed-transcribed using a T7oligo(dT) promoter-primer in the first-strand DNA-synthesis reaction. Following RNase H-mediated secondstrand cDNA synthesis, the double-stranded cDNA was purified and used as a template for the subsequent in-vitro transcription reaction. This reaction was carried out in the presence of T7-RNA polymerase and a biotinylated nucleotide analogue/ribonucleotide mix for cRNA amplification and biotin labeling. The biotinylated cRNA targets were then cleaned up, fragmented, and hybridized to the GeneChip expression array. The chip was reacted with streptavidin-phycoerythrin and then with biotinylated anti-streptavidin antibody (Vector Laboratories, Burlingame, CA). Arrays were scanned by GeneArray scanner G2500A (Hewlett Packard, Palo Alto, CA), visually inspected for hybridization imperfections and analyzed using Affymetrix Microarray Suite software version 5.0 by scaling to an average intensity of 250 . The raw data have been deposited in the public repository "Gene Expression Omnibus" (GEO), accession no. GSE15119.

\section{Statistical, hierarchical clustering and functional annotation analyses}

The data were analyzed with GeneSpring (Silicon Genetics, Redwood City, CA) using the MAS5 algorithm [25]. Gene-expression data were normalized "per chip" and "per gene". For "per chip" normalization, all expression data on a chip were normalized to the $50^{\text {th }}$ percentile of the measurements taken from all values on that chip. For "per gene" normalization, each gene's measurement in the selected samples was divided by the median of the gene's measurements in the respective control group, according to the type of comparison being made. When profiles were compared between tumors induced by each of the two transgenic Stat5 variants, the expression of a given gene was normalized to the median of the expression level in the wild-type mammary gland samples. This allowed relating the level of expression of a given gene to that in the mammary gland [23]. When the analysis also included values obtained from the mammary glands, the expression of a given gene was normalized to the median of the expression levels of all genes from all samples. The normalized data were log-transformed and the differences in gene expression (based on the individual values obtained from each tumor) were calculated using oneway statistical analysis of variance (ANOVA). The statistical analysis, which discriminates between the effects of transgenic STAT5 variants, was cross-validated by the Knearest-neighbor algorithm using the leave-one-out methodology [26]. The differences in gene expression between the tumors and the mammary gland were determined by post-hoc Student-Newman- Keuls analysis. Note that the different expression patterns of selected genes in the array had been previously confirmed by semi-quantitative PCR analyses of selected genes, and hybridization to the respective probes [23].

Genes exhibiting a significant $(P \leq 0.05)$ twofold difference in expression between transgenic variants were categorized into "biological functions and/or diseases" using Ingenuity Pathways Analysis (IPA) software (Ingenuity Systems, Mountain View, CA). The probability of each term being identified by random chance was calculated using Fisher's exact test http://www.ingenuity.com/. Unsupervised hierarchical clustering (GeneSpring) organized these genes according to their similarity or dissimilarity in expression profiles, placing the cases with similar expression profiles together as neighboring rows in the clustergram.

Multivariate correlations were determined between the expressions of transgenic STAT5 and specifically affected genes, and among the expressions of the affected genes in the individual tumors using JMP statistical software (Version 7.0, SAS Institute, Inc., Cary, NC).

\section{Real-time PCR}

Quantitative real-time PCR analyses were performed in an ABI Prism 7700 (Applied Biosystems, Foster City, CA) in a 20- $\mu \mathrm{l}$ reaction volume containing $4 \mu \mathrm{l}$ cDNA (diluted 1:100), $10 \mu \mathrm{l}$ SYBR Green PCR Master Mix (Applied Biosystems) and $10 \mu \mathrm{M}$ primers. The thermal-cycle conditions consisted of $2 \mathrm{~min}$ at $50^{\circ} \mathrm{C}, 2 \mathrm{~min}$ at $95^{\circ} \mathrm{C}$, and 40 
cycles of $15 \mathrm{~s}$ at $95^{\circ} \mathrm{C}$ and $1 \mathrm{~min}$ at $60^{\circ} \mathrm{C}$. The primers were designed so that the PCR would yield a single product without any primer dimerization, and the product was verified using a dissociation protocol [see Additional file 1 , data sheet A]. The primers were designed across exonexon junctions to ensure that there was no genomic DNA contamination in the cDNA samples. The amplification curves for the selected genes (70-100 bp) were parallel.

\section{Results \\ Establishment of distinct expression profiles for the effects of STAT5ca and STAT5 750 in mammary tumors}

RNA was independently extracted from six tumors developed in mice carrying the BLG/STAT5 $\Delta 750$ transgene and from seven tumors originating in mice carrying BLG/ STAT5ca. Tumor phenotypes were either poorly differentiated carcinoma or papillary adenocarcinoma (Fig. 1 and described in [15]). Following microarry hybridization, we identified a set of 381 features (364 genes) exhibiting a significant $(P<0.05)$ twofold difference in expression level between tumors originated from mice carrying the two transgenic STAT5 variants [see Additional file 1, data sheet B]. Their grouping according to type of transgenic STAT5 variant was confirmed by principal component analysis (PCA, [27]) and unsupervised hierarchical clustering (Fig. 1).

IPA software was used to annotate the differentially expressed genes into processes involved in cellular metabolism and cancer (Table 1). In both categories, the highest number of differentially expressed genes in the STAT5cavs. STAT5 $\Delta 750$-induced tumors was associated with two opposing processes: cell proliferation and cell death [see Additional file 1, data sheet $\mathrm{C}$ ]. The involvement of some of the genes affecting cancer cell death-Adm, Cflar, Cyr61, Ddit4, Itgb1, Mapk1, Mapk8, Tgf 32 , Tpm1, Vegf $\alpha$ and Wasf1, was demonstrated in breast cancer cell lines [see Additional file 1, data sheet $\mathrm{C}$. The activities of Itgb1 (coding for integrin $\beta 1$ ), TGF $\beta 2$ and Vegf $\alpha$ are mediated, at least in part, by Cyr61 (CCN1, [28-30])-a secreted matrix protein involved in the clinical progression of breast cancer to an invasive phenotype [31].

Cyr61 can be also found among the differentially expressed genes regulating the formation of cellular protrusions and filopodia-cellular extensions needed for cellular interactions and movement [32]. Most of the listed genes affecting this process, including the laminin $\alpha 5$ (Lama5) [33] and the VEGF receptor PVR [34], were expressed at higher levels in the STAT5 $\Delta 750$-induced tumors. This suggests higher involvement of this variant in cell movement and tumor extension. The GDP/GTP exchange protein Fabrin 4 (FDG4, [34] and the ganglioside GD3-synthase (ST8SIA)1 which marks ER-negative breast cancer tumors [35] were the only genes in this con- text that were expressed at a higher level in the STAT5cainduced tumors.

The differential expression of genes mediating carbohydrate metabolism indicates involvement of the STAT5 variants in cellular homeostasis as well. Most of the genes involved in the transport and utilization of carbohydrates were more highly expressed in tumors caused by the BLG/ STAT5 4750 transgene, for example, Aqp7 and Aqp9 which code for proteins operating as glycerol channels [36], or glucokinase (Gck) which encodes a protein that catalyzes the conversion of glucose to glucose-6-phosphate, thus maintaining glucose homeostasis [32].

\section{Hierarchical clustering of genes affected by transgenic STAT5 variants}

Unsupervised hierarchical clustering assembled the genes that were differentially expressed between the two sets of STAT5-affected tumors into four clusters (Fig. 2 and [see Additional file 1, data sheet D]). Cluster 1 contains genes that were more highly expressed in the STAT5ca-induced tumors than in their STAT5 $\Delta 750$ counterparts, resulting from downregulation of the STAT $5 \Delta 750$-induced genes compared to their expression in the mammary gland (represented by the value " 1 "). In contrast, genes allocated to clusters 2, 3 and 4 were more highly expressed in the STAT5 $\Delta 750$-induced tumors. In cluster 2 , the STAT $5 \Delta 750$ affected genes were, in general, also more highly expressed than in the wild-type gland. In cluster 3 , the STAT5 $\Delta 750$ affected genes maintained mammary gland levels of expression while their STAT5ca counterparts were expressed at a lower level. Finally, in cluster 4, differential expression relative to the mammary gland was noted: higher expression of genes induced by the BLG/ STAT5 $\Delta 750$ transgene, lower expression of genes affected by its BLG/STAT5ca counterpart.

Annotation of the 76 genes in cluster 1 assembled them into non-malignant cellular processes, including amino acid modification, hematopoiesis and neurological disorders [see Additional file 1, data sheet E]. In contrast, all other clusters which included genes expressed at higher levels in the STAT5 $\Delta 750$-induced tumors contained the "cancer" category which assembled genes into specific tumorigenic processes. In this category, genes mediating "invasion", such as Adm, Itg3 and Igf2, converged to cluster 2 . In contrast, most genes involved in tumor growth, transformation and differentiation were expressed at lower levels and were therefore allocated to cluster 3.

\section{Transgenic STAT5 variants exert distinct effects on a limited set of specifically affected genes}

Specifically affected genes differed in their expression between an individual tumor set and its counterpart, and between that set and the intact mammary gland. To iden- 

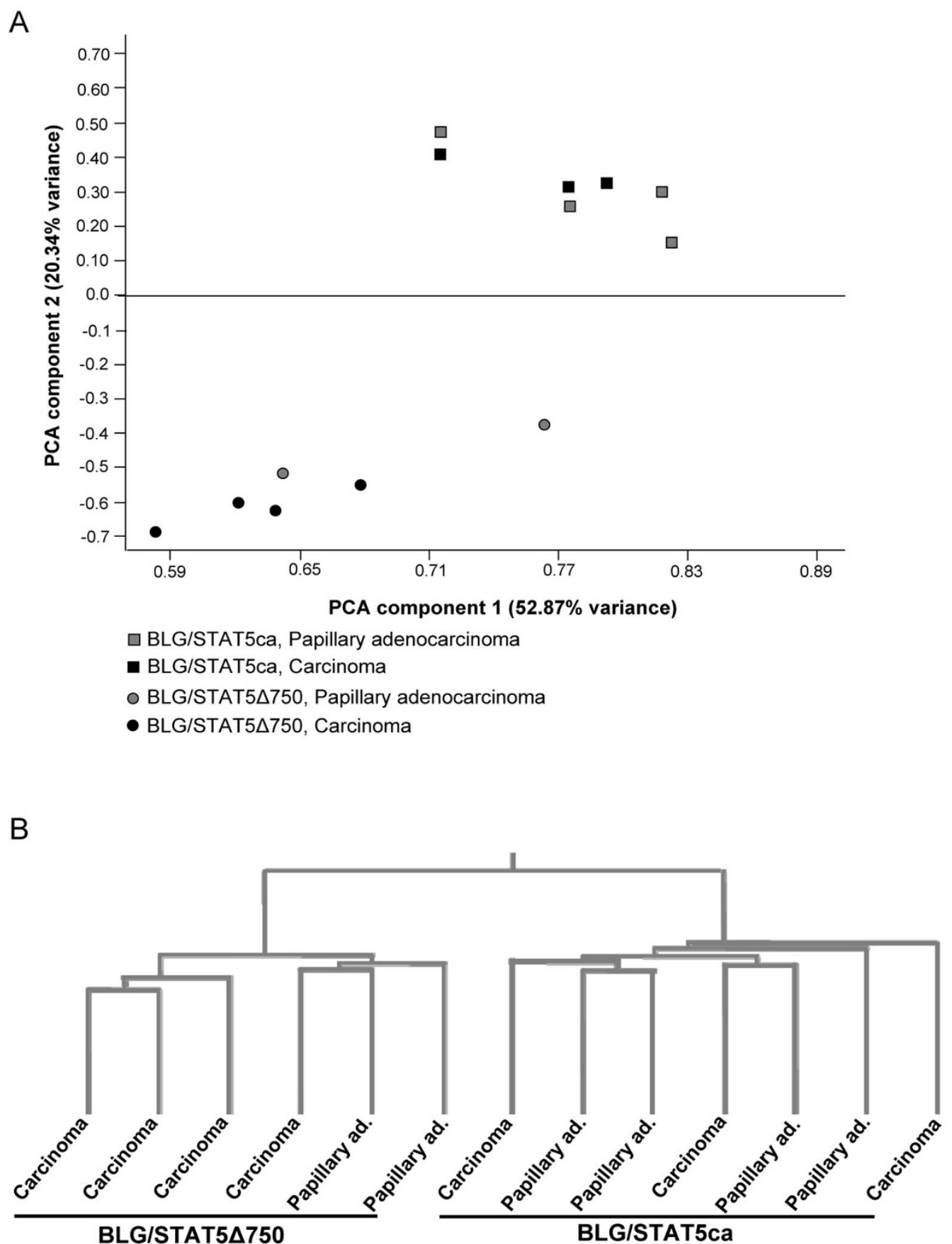

Figure I

Principal component analysis (PCA) and unsupervised hierarchical clustering into distinct tumor genotypes. Mammary carcinomas and papillary adenocarcinomas were developed in transgenic mice expressing constitutively activated STAT5 (STAT5ca) or truncated STAT5 (STAT5 4750$)$. PCA (A) and unsupervised hierarchical clustering (standard correlation, $B)$ were performed on genes that were expressed at significantly $(P<0.05$; twofold) different levels in tumors induced by the two STAT5 variants. Both analyses confirmed their distinction based on the transgenic STAT5 variant carried by the host female. 
Table I: Cellular metabolism and cancer processes affected by genes that are differentially expressed in tumors caused by the different transgenic STAT5 variants.

\begin{tabular}{|c|c|c|c|c|}
\hline Category & Process & Process Annotation & Significance & No. of Molecules \\
\hline \multirow[t]{4}{*}{ Carbohydrate Metabolism } & formation & formation of inositol phosphate & $1.53-2$ & 3 \\
\hline & production & production of carbohydrate & $8.32^{-3}$ & 7 \\
\hline & transport & transport of carbohydrate & $1.66^{-5}$ & 11 \\
\hline & utilization & utilization of carbohydrate & $1.04-2$ & 3 \\
\hline \multirow{2}{*}{$\begin{array}{l}\text { Cardiovascular System Development and } \\
\text { Function }\end{array}$} & angiogenesis & angiogenesis of cells & $1.69-3$ & 5 \\
\hline & cardiovascular process & cardiovascular process of cornea & $6.15^{-4}$ & 6 \\
\hline \multirow[t]{5}{*}{ Cell cycle } & GI phase & GI phase of eukaryotic cells & $1.88^{-2}$ & 11 \\
\hline & G2 phase & G2 phase of tumor cell lines & $1.59-2$ & 5 \\
\hline & interphase & interphase of eukaryotic cells & $1.36^{-2}$ & 17 \\
\hline & length & length of telomeres & $1.72^{-2}$ & 3 \\
\hline & mitogenesis & mitogenesis & $3.30^{-3}$ & 10 \\
\hline \multirow[t]{3}{*}{ Cell death } & cell death & cell death & $1.43^{-3}$ & 74 \\
\hline & cell death & cell death of cell lines & $5.03^{-4}$ & 49 \\
\hline & cell death & cell death of tumor cell lines & $2.11-4$ & 41 \\
\hline \multirow{4}{*}{ Cellular Assembly and Organization } & formation & formation of cellular protrusions & $1.18^{-2}$ & 7 \\
\hline & & formation of filopodia & $7.48^{-4}$ & 8 \\
\hline & & $\begin{array}{l}\text { formation of plasma membrane } \\
\text { projections }\end{array}$ & $1.77^{-3}$ & 13 \\
\hline & growth & $\begin{array}{l}\text { growth of plasma membrane } \\
\text { projections }\end{array}$ & $1.21^{-2}$ & 13 \\
\hline \multirow[t]{3}{*}{ Cellular Growth and Proliferation } & formation & formation of eukaryotic cells & $2.93^{-3}$ & 13 \\
\hline & growth & growth of cell lines & $4.4 I^{-4}$ & 36 \\
\hline & proliferation & proliferation of eukaryotic cells & $1.58^{-6}$ & 65 \\
\hline \multirow[t]{4}{*}{ Cellular Movement } & cell movement & cell movement of eukaryotic cells & $1.25^{-3}$ & 45 \\
\hline & & cell movement of tumor cell lines & $1.23-4$ & 22 \\
\hline & homing & homing of eukaryotic cells & $5.69-3$ & 17 \\
\hline & migration & migration of tumor cell lines & $2.23^{-4}$ & 17 \\
\hline $\begin{array}{l}\text { DNA Replication, Recombination and } \\
\text { Repair }\end{array}$ & synthesis & synthesis of DNA & $3.78^{-3}$ & 17 \\
\hline $\begin{array}{l}\text { Hematological System Development and } \\
\text { Function }\end{array}$ & hematological process & hematological process & $7.64^{-3}$ & 24 \\
\hline $\begin{array}{l}\text { Nervous System Development and } \\
\text { Function }\end{array}$ & growth & growth of neurites & $1.02-2$ & 13 \\
\hline Tissue Morphology & contraction & contraction of tissue & $6.01^{-4}$ & 12 \\
\hline \multirow[t]{11}{*}{ Cancer } & benign tumor & benign tumor & $1.55^{-2}$ & 10 \\
\hline & carcinoma in situ & carcinoma in situ & $1.77-2$ & 6 \\
\hline & apoptosis & apoptosis of tumor cell lines & $2.33^{-4}$ & 37 \\
\hline & cell death & cell death of tumor cell lines & $\left.2.1\right|^{-4}$ & 41 \\
\hline & & cell death of breast cancer cell lines & $1.12^{-2}$ & II \\
\hline & cell movement & cell movement of tumor cell lines & $1.23^{-4}$ & 22 \\
\hline & growth & growth of tumor & $2.03^{-3}$ & 10 \\
\hline & & growth of tumor cell lines & $1.01-2$ & 25 \\
\hline & metastasis & migration of tumor cell lines & $2.23^{-4}$ & 17 \\
\hline & proliferation & proliferation of tumor cell lines & $1.29-4$ & 28 \\
\hline & transformation & transformation of cells & $1.31-2$ & 19 \\
\hline
\end{tabular}


tify genes which were specifically affected by each of the STAT5 variants, three mammary gland samples were added to the analysis, and the level of expression of the 364 genes exhibiting differential expression among STAT5-induced tumors was re-evaluated (Fig. 3). This analysis identified 52 genes associated with the effect of the STAT5 $\Delta 750$ transgene $[($ STAT5 $\Delta 750 \neq$ STAT5 $\mathrm{ca}=$ mammary gland (M.G.)], 50 genes that were specifically affected by STAT5ca (STAT5ca $\neq$ STAT5 $\Delta 750=$ M.G.), and 62 genes with differential expression in the three groups (STAT5 $\Delta 750 \neq$ STAT5 $\mathrm{ca} \neq$ M.G., and [see Additional file 1, data sheet F]). Unexpectedly, 94\% of the STAT5ca-affected genes (47 genes) were downregulated compared to their expression in the STAT5 $\Delta 750$-induced tumors and the wild-type mammary gland. A considerable number of these genes could be associated with anti-proliferative or tumor-suppressive processes, including apoptosis inducers Edg3 and Jip-2 [37,38], differentiation inducers Msln, Il5ra and Ebf2 [39-41], Prp19 (also known as Pso4) which is involved in DNA repair [42], Ragef3 which blocks chemotaxis induced by angiogenic factors [43] and Tyrp, which is expressed in poorly metastatic breast cancer and whose downregulation augments metastasis [44].

A more equal deviation characterized the STAT5 $\Delta 750$ affected genes. Only $38 \%$ of these genes were downregulated, whereas the rest were expressed at significantly higher levels compared to their expression in the STAT5ca-induced tumors or the wild-type mammary gland. In contrast to the STAT5ca-affected genes that were located in cluster 3, their STAT5 $\Delta 750$ counterparts spread mainly among cluster 1 (20 genes, 38\%) and cluster 2 (29 genes, $56 \%$ ), with a residual presence in cluster 4 ( 3 genes, $6 \%)$. Upregulated and downregulated genes were annotated with different activities (Table 2). Upregulated genes affected angiogenesis, cell adhesion, cell-cell signaling, and progression through the cell cycle. Downregulated genes were involved in insulin receptor binding, DNA replication, chromatin remodeling and G-protein receptor pathways.

\section{The expression of STAT5ca in individual tumors does not correlate with the expression of its specifically affected genes}

Does the deviation in gene expression between the two sets of tumors result from an early transgenic STAT5 effect, or does continuously deregulated STAT5 expression in the tumors govern the gene-expression profile?

STAT5ca and STAT $\Delta 750$ expression levels were analyzed by real-time PCR in the respective tumors. In contrast to measurable STAT5ca transcripts, STAT5 450 mRNA did not reach detection levels. There was no significant correlation between expression of STAT5ca in the tumors and that of any of its affected genes [see additional file 2, bot- tom row of upper section]. Interestingly, within the genes affected specifically by STAT5ca, a few groups were identified which were linked by significant correlations between the expressions of their individual members. The genes with the highest number of internal correlations are presented [see Additional file 2]. The largest such group included Mapk8 (top row), Wnt8b, Ptn, Pramel6, Klra5, Edg3, Ebf2 and Tyrp1. Significant gene-expression correlations were also detected among the STAT5 $\Delta 750$-affected tumors, the largest group including Wnt7b, Scgb1a1, Itga3, Mrpplf4 and Cspg2. Analyses of these gene groups by DAVID [45] and IPA software could not assemble the individual members into a single metabolic pathway or identify a mutual effector.

Distinct distribution into clusters was observed when the specifically affected genes were allocated. With the list of highly correlated genes as a core, the STAT5ca-affected genes were assembled into a small region in the upper section of cluster 3 (Fig. 2), confirming a lower level of expression compared to both the STAT5 $\Delta 750$-induced tumors and the wild-type mammary gland. In contrast, genes affected by STAT $\Delta \Delta 750$ were distinctly located in clusters 2 and 1 . Their location in cluster 2 implied a higher level of expression compared to those specifically affected by the STAT5ca transgene or the wild-type mammary gland. Their presence in cluster 1 as well reflected the more even distribution between upregulated and downregulated genes.

\section{Reproducing transgenic STAT5 effects in a "test set" of tumors}

Our next step was to confirm the "transgenic signature" of the STAT5 variants in independent groups of tumors. From the list of 50 genes specifically affected by STAT5ca, six candidates were selected and their expression was analyzed by real-time-PCR in a separate set of tumors caused by the two STAT5 variants. This "test set" included seven tumors that had developed in BLG/STAT5ca-transgenic mice (four papillary adenocarcinomas, one squamous carcinoma and two poorly differentiated carcinomas) and six tumors that had developed in the BLG/STAT5 $\Delta 750$ mice (one papillary adenocarcinoma, three micropapillary adenocarcinomas, one squamous carcinoma and one adenosquamous carcinoma). As demonstrated in Table 3, the differences between the expressions of genes affected by STAT $5 \Delta 750$ vs. STAT5ca detected in the original set of tumors that was tested by the array analysis were comparable to those found in the "test set" which was subjected to real-time PCR.

\section{Discussion}

The constitutively active Stat 5 and its C-terminally truncated variant have been implicated in cancer in laboratory animals and humans. Here we established a model sys- 

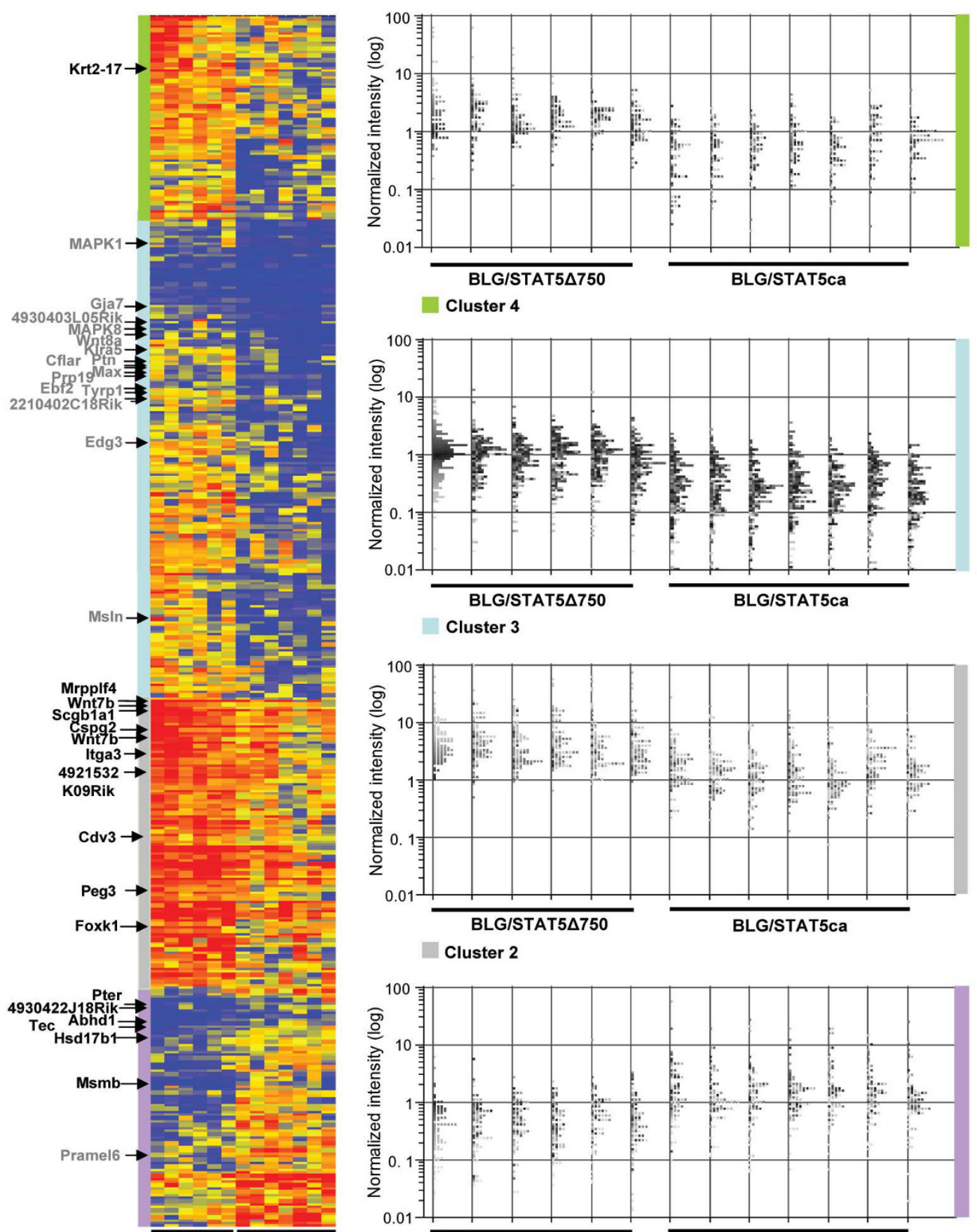

Cluster 3

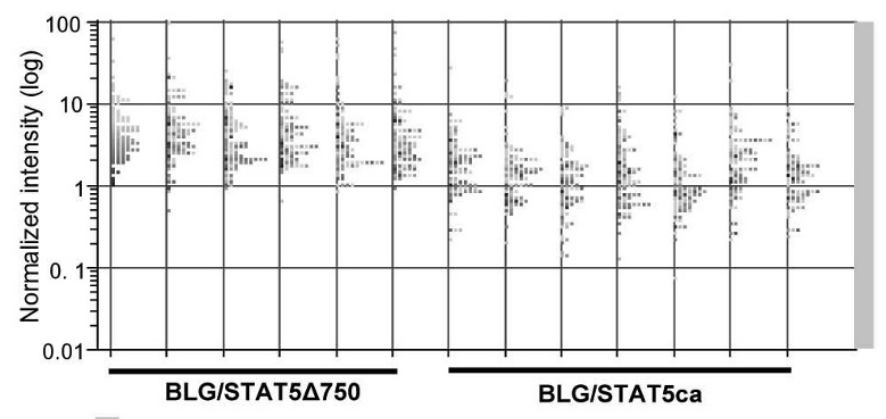

Cluster 2

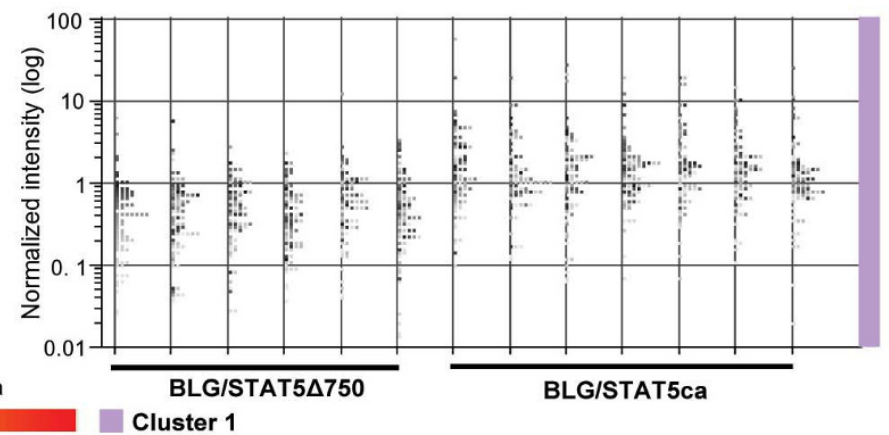

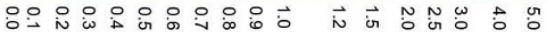

Level of expression

Figure 2

Unsupervised hierarchical clustering of genes that are differentially expressed in tumors induced by STAT5ca and STAT5 $\triangle 750$ transgenes. Genes with statistically significant $(P<0.05)$, over twofold differences in expression levels between the two sets of tumors were clustered. In each cluster, cellular processes were annotated. The normalized intensity values indicate relative expression levels compared to the median gene expression in the intact mammary gland ("I"). Arrows mark genes that are specifically affected by STAT5ca (gray) or STAT5 $\triangle 750$ (black) and listed in Table 2. 
A

STAT5 $\Delta 750$ vs. STAT5ca in tumors

$P \leq 0.05, \geq$ two fold change

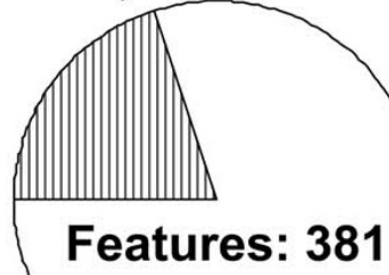

Genes: 364

B

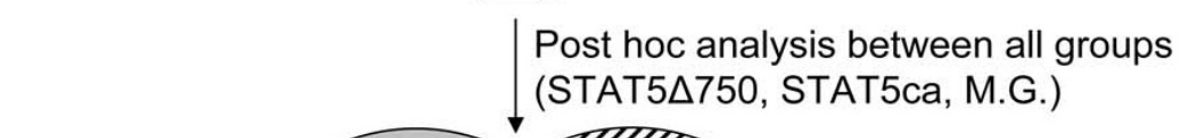

C
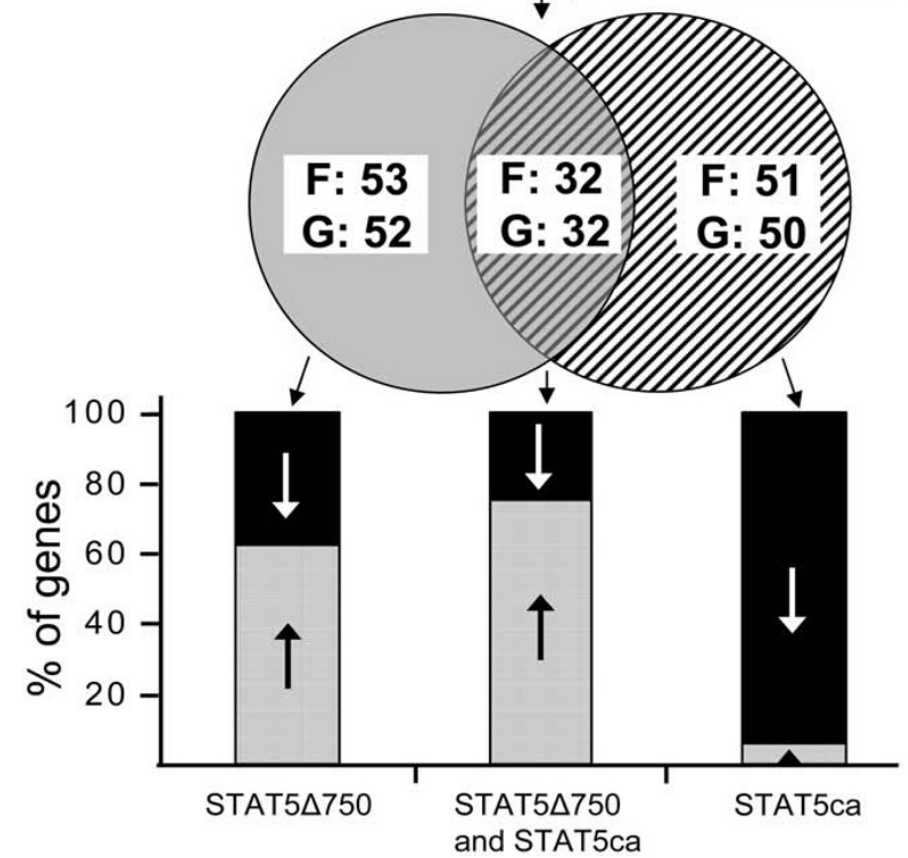

Higher expression in STAT5 $\Delta 750$ tumors

孟 Higher expression in STAT5ca tumors

Specifically affected by STAT $5 \Delta 750$

$\mathbb{Z}$ Differentially expressed between STAT5 $\Delta 750$, STAT5ca and M.G.

Q Specifically affected by STAT5ca

4 Upregulated genes

† Downregulated genes

Figure 3

Venn Diagrams of genes affected by the transgenic STAT5 variants relative to their expression in the mammary gland. A. Levels of expression in the two sets of tumors were compared. B. Mammary gland expression levels were included in the analysis to identify genes that differ in their expression in a particular group (specifically affected genes). C. Upor downregulation of gene expression in individual groups was defined. 
Table 2: Classification, annotation and expression levels of genes, specifically affected by the STAT5 variants.

\begin{tabular}{|c|c|}
\hline Specifically affected by BLG/STAT5ca & \\
\hline Ras small GTPase & Rasd I, Rasd2 \\
\hline GTPase activity & RasdI Rasd2 GNAOI \\
\hline Rho GAPs & Chnl \\
\hline G-protein & GNAOI \\
\hline G-protein-coupled receptor & Edg3, Adra2c \\
\hline Cytoskeleton protein & Tnnil, Cnnl, Rsnl2 \\
\hline GEFs & Rapgef3 \\
\hline Cytoskeleton rearrangement & Ptn, pip5klb \\
\hline Organization of microtubules & Rsnl2 \\
\hline Cell-cell recognition/signaling/adhesion & Msln, ptn, Rapgef3, Adra2c \\
\hline Cell migration & Edg3 \\
\hline Mammary development & Ebf2, Gli2 \\
\hline Immune system/response & Csf2ra, il5ra, Foxji, H2-KI, EDG3 \\
\hline Neurons & Elavl3, Wnt8a, Gfra2, Phgdh \\
\hline DNA damage response/cell cycle & Prp 19 (DDR), Magea2 (inhibits p53 activation) \\
\hline Apoptosis inhibition & Cflar \\
\hline Tight junction & Mpdz, Gja7 \\
\hline Potassium channel & Kcne4 \\
\hline JNK signaling & JIP-2, Rapgef3, Mapk8 \\
\hline Transcription factor & Max \\
\hline Defense response & KIra5, KIra22 \\
\hline Ribosome & Rps I5a \\
\hline Urea cycle & Otc \\
\hline Signal transduction & $\begin{array}{l}\text { MapkI, JIP-2, Rapgef3, Mapk8, Gfra2, Adra2c, RasdI, Rasd2, GNAOI, } \\
\text { Chn I, EDG3, Wnt8a, JIP-2 }\end{array}$ \\
\hline Protein phosphates inhibitor activity & PPPIR2P9 \\
\hline Regulation of nucleotide and nucleic acid metabolism & Ddx3y \\
\hline Bacterial cell wall degradation & lysmd2 \\
\hline Regulating hemoglobin oxygen affinity & Bpgm \\
\hline Expressed in the early embryo & Pramel6 \\
\hline Not associated & $\begin{array}{l}\text { TyrpI, I700065II7Rik, Serpinb6c, 20 I0003K IIRik, 493 I4I2G03Rik } \\
\text { (Trpd52I3) }\end{array}$ \\
\hline
\end{tabular}

Specifically affected by BLG/STAT5 $\Delta 750$

\begin{tabular}{|c|c|}
\hline Adm, Mrpplf4, Cyr6I & Angiogenesis \\
\hline Cspg2, Itg $\beta$ I, Itg $\beta 3$, Scgb I I, Pcdhb5, Rin I, Lana5, Cyr6I, Cav2 & Cell adhesion \\
\hline Itg $\beta$ I, Foxk I, RnfI34, Peg3, Mkrn3 & Progression through cell cycle \\
\hline Peg3, Kif5c, Pcdhb5, Ivns I abp, Mkrn3 & Neuron \\
\hline Foxkl, Rnfl34, Peg3 & Transcription factor activity \\
\hline Gdi2, Rin I & GTPase activator activity \\
\hline Tec, Trhr, Oprl, VIra9, 59304I8KI5Rik & G-protein-coupled receptor protein signaling pathway \\
\hline Adm, Wnt7b, Pcdhb5 & Cell-cell signaling \\
\hline Irs4 & Insulin receptor binding \\
\hline Orc3l & DNA replication \\
\hline Eif2s2 & Translation initiation factor activity \\
\hline Cugbpl & Translation repressor activity \\
\hline Cdv3, Cyr6I & Cell proliferation \\
\hline HsdI7bl & Estradiol 17-beta-dehydrogenase activity \\
\hline H2-EbI, Tec, Igbp Ib, Nudcd I & Immune response \\
\hline Nudcd I, Mkrn3, Msmb, Cav2, Gnmt, Cryll & Cancer-related \\
\hline Kif5c & Microtubule-based movement \\
\hline Abhdl & Hydrolase activity \\
\hline KIfI & Chromatin remodeling \\
\hline Peg3, Mkrn3 & Apoptosis \\
\hline B3galt I & Transferase activity, transferring glycosyl groups \\
\hline Igbp Ib & B-cell activation \\
\hline Krt2-I7, Kif5c & Cytoskeleton organization and biogenesis \\
\hline Pdss I & Isoprenoid biosynthetic process \\
\hline
\end{tabular}


Table 2: Classification, annotation and expression levels of genes, specifically affected by the STAT5 variants. (Continued)

\begin{tabular}{ll}
\hline Pi4k2b & Phosphotransferase activity, alcohol group as acceptor \\
Nol5 & Ribosome biogenesis and assembly \\
Fabp5 & PPAR signaling pathway/lipid metabolic process \\
Gnmt & Breaks down the protein building block (amino acid) methionine \\
$4930422 J 18 R i k(S S B-1)$ & Ubiquitin cycle \\
C80 I 1 I, 1810030007Rik, Es2el, Pter & Not associated
\end{tabular}

In black-genes with higher expression in tumors induced by STAT5 $\Delta 750$. In gray-genes with higher expression in tumors induced by STAT5ca.

tem, based on mice expressing the individual transgenic STAT5 variants, to study the distinct variants' oncogenic role in the mammary gland.

Stat5 mediates proliferation in the mammary gland of pregnant females [46], and different effects of its two variants have been demonstrated in transgenic mice during the reproductive cycle: STAT5ca-induced proliferation during pregnancy and delayed apoptosis and tissue remodeling during involution [11]. The opposite has been shown for the STAT5 $\Delta 750$ variant $[11,12]$. This diversity results from the structural differences between the STAT5 variants which involves either forced activation or lack of TAD-mediated interactions with the proteins, such as CBP/p300, that recruit acetylases [47]. Both STAT5 variants seem to have a persistent effect, probably on chromatin structure and accessibility $[16,48,49]$, which results in different profiles of gene expression in the developing tumors.

Differential expression of 364 genes was found between mammary tumors developed in BLG/STAT5ca- and BLG/ STAT5 $\Delta 750$-transgenic mice. These genes were involved in a set of cellular activities, many of which could still be associated with the two main processes mediated by Stat 5 in the intact tissue: cell proliferation and cell death. The individual genes that established the specific mark of the STAT5 variants in the tumors were identified by genearray analysis and their differential expression in the population was confirmed in a distinct "test set" of tumors using a different detection method, real-time PCR. Of spe- cial interest was the gene encoding the proto-oncogene Met, which was expressed in the STAT5 $\Delta 750$-induced tumors at a 22-fold higher level than in their STAT5cainduced counterparts. The product of Met is the hepatocyte growth factor receptor which encodes tyrosine-kinase activity. The ligand-activated cytoplasmic domain of the cMet receptor induces growth motility, morphogenesis and angiogenesis [50]. In breast cancer, c-Met overexpression is associated with tumor progression (reviewed in [51]) and has an independent predictive value for poor survival, even in early-stage patients with negative lymph nodes [52]. Expression levels of Met were upregulated relative to intact tissue in the STAT5 $\Delta 750$-induced tumors and downregulated in tumors developed in mice carrying the BLG/STAT5ca transgene. This differential expression suggests a more aggressive downstream cascade in the former. Other genes with exceptionally high expression levels in the STAT5 $\Delta 750$-induced tumors, which were not affected by the STAT5ca variant, were proliferin and Igf2. Proliferin is a member of the prolactin family which is involved in progenitor cell expansion along the luminal and myoepithelial lineage [53] and Igf2 plays a pivotal role in fetal and cancer development by signaling via the IGF-I and insulin receptors, and activating the estrogen-signaling cascade [54]. Unfortunately, studies on these genes [5557] do not include or base additional lists of genes with altered expression profiles that might be compared to our data and aid in delineating the pathway(s) involved in the tumorigenic effect of STAT5 $\Delta 750$.

Table 3: Validation of the differences in gene expression determined by the array analysis using real-time PCR of gene expression in a test set of tumors.

\begin{tabular}{|c|c|c|c|c|c|c|}
\hline \multirow[t]{2}{*}{ Gene } & \multicolumn{3}{|c|}{$\begin{array}{c}\text { Tumor set I } \\
\text { (Microarray analysis, arbitrary units) }\end{array}$} & \multicolumn{3}{|c|}{$\begin{array}{c}\text { Tumor set } 2 \text { (test set) } \\
\left.\text { (Real-Time PCR analysis, } 2^{-\Delta c t} \times 1,000\right)\end{array}$} \\
\hline & $\begin{array}{c}\text { BLG/STAT5 } \Delta 750 \\
\text { tumors }\end{array}$ & $\begin{array}{l}\text { BLG/STAT5ca } \\
\text { tumors }\end{array}$ & $\begin{array}{c}\text { Fold change (BLG/ } \\
\text { STAT5 } \triangle 750 / B L G / \\
\text { STAT5ca) }\end{array}$ & $\begin{array}{c}\text { BLG/STAT5 } \Delta 750 \\
\text { tumors }\end{array}$ & $\begin{array}{l}\text { BLG/STAT5ca } \\
\text { tumors }\end{array}$ & $\begin{array}{c}\text { Fold change (BLG/ } \\
\text { STAT5 } \Delta 750 / B L G / \\
\text { STAT5ca) }\end{array}$ \\
\hline Edg3 & $1.59 \pm 0.21$ & $0.43 \pm 0.15$ & 3.64 & $0.10 \pm 0.02$ & $0.04 \pm 0.01$ & 2.08 \\
\hline Mapk8 & $1.30 \pm 0.19$ & $0.51 \pm 0.12$ & 2.54 & $0.64 \pm 0.189$ & $0.43 \pm .05$ & 1.49 \\
\hline Wnt8a & $|.4| \pm 0.3 \mid$ & $0.44 \pm 0.14$ & 3.15 & $1.41 \pm 0.380$ & $0.37 \pm 0.21$ & 3.75 \\
\hline Ptn & $1.69 \pm 0.48$ & $0.46 \pm 0.25$ & 3.68 & $1.01 \pm 0.219$ & $0.48 \pm 0.07$ & 2.09 \\
\hline Foxkl & $3.49 \pm 0.97$ & $0.86 \pm 0.19$ & 4.04 & $0.35 \pm 0.118$ & $0.25 \pm 0.02$ & 1.42 \\
\hline Tyrpl & $1.37 \pm 0.21$ & $0.41 \pm 0.17$ & 3.33 & $2.22 \pm 1.159$ & $0.47 \pm 0.18$ & 4.71 \\
\hline
\end{tabular}


A group of genes regulating carbohydrate metabolism and transport could also be discerned. These genes were not associated with Stat 5 effects in the intact mammary gland but were differentially expressed in the two sets of tumors. Their role in the tumors could be related to altered levels of metabolism (i.e. higher metabolic rate in the STAT5 $\Delta 750$-induced tumors) rather than cancer growth per se.

Expression of an additional set of 14 genes linked the effects of the BLG/STAT $5 \Delta 750$ and the BLG/STAT5ca transgenes to the resulting phenotypes of poorly differentiated carcinoma or highly differentiated papillary adenocarcinoma, respectively $(\mathrm{r}=0.97)$ [see Additional file 1 , data sheet $G$ and [16]]. These genes cover a wide variety of cellular functions: calcium sensitivity of the myofibrils (troponin I and tropomyosin 1), interaction between the cell and the extracellular matrix (endomucin), normal adipose tissue development (lipin 3), lipid metabolism, cellular growth and apoptosis (caveolin 2), mRNA metabolism (5'-3' exoribonuclease 1), mitochondrial fatty acid oxidation (acyl coenzyme A dehydrogenase), as well as kinase and protease activities (TAU tubulin kinase 1 and corin, respectively). Taken together, their diverse expression is most likely involved in determining tumor phenotype, i.e. associating higher proportions of the poorly differentiated carcinomas or the highly differentiated papillary adenocarcinomas with the expression of STAT5 $\Delta 750$ or STAT5ca, respectively [15].

Overall, 94\% of the genes specifically affected by STAT5ca were downregulated relative to their expression in the host tissue. This contrasts with the more equal specific effect of the STAT5 $\Delta 750$ variant and provides additional evidence for the distinct routes via which the two STAT5 variants impose their mark on tumor growth and maintenance. The high number of tumor and growth suppressors defined among the STAT5ca-downregulated genes vs. the potent oncogenes (Met, Igf2) that were induced by the STAT5 $\Delta 750$ variant may serve to further distinguish the routes via which they initiate and maintain tumorigenesis.

Substantial downregulation of gene expression has been demonstrated in breast cancers with bone marrow (BM) micrometastasis [58], and during the molecular transition from organ-confined to metastatic prostate cancer [59]. Apparently, transcription repression is important for the metastatic process in these tissues. When compared with the list of genes downregulated by STAT5ca-, no overlap was observed for those associated with BM micrometastasis, and only four genes (Ptn, Cflar, Cnn1 and Mpdz) shared the list of downregulated genes mediating prostate metastasis. Thus, the resultant tumor characteristics are probably determined by a combination of the phenome- non of gene downregulation per se and the properties of the specifically affected genes (in this case by STAT5ca).

Our attempt to characterize the different roles of the STAT5 variants in mammary cancer development did not produce any evidence for the effect of transgenic STAT5 expression in the tumors. Neither distinct metabolic pathways nor a central mediator(s) were located. This suggests domination of an earlier STAT5 effect on the resulting gene-expression profiles. Several groups of genes, each composed of three to eight members, with significant internal correlations were identified among the sets specifically affected by STAT5ca and STAT $5 \Delta 750$. This correlation was not associated with physical linkage. Within a single group, genes with correlated expression might control a distinct range of cellular functions such as angiogenesis (Ptn and Tyrp1 [60,61]), apoptosis (Cflar, [62]) and morphogenesis (Ebf2, [63]). Their comparable levels of expression, defined by their location in a confined region within the cluster, suggest that although these genes are mapped to several loci, they may colocalize to a shared transcription site. The concept of several loci being targeted to a shared transcription site where they generate "transcription factories" with similar levels of expression was proposed a few years ago and is reviewed in $[64,65]$. The relevance of this biological system to the specific STAT5 effects presented in this study remains to be determined. Regardless of the detailed mechanism involved in the effect of STAT5 $\triangle 750$ and STAT5ca on tumor growth and maintenance, this study establishes the feasibility of identifying and distinguishing mammary tumors according the variants' signature on specific gene-expression profiles. To the best of our knowledge, this signature is specific to the effect of the STAT5 variants. However, its uniqueness will only be confirmed by the contribution of further gene-expression profiles that are specific for the effects of other oncogenes.

\section{Conclusion}

The C-terminally truncated form of Stat 5 and its constitutively active variant retain oncogenic potency that is conveyed via distinct pathways and probably initiated during early stages of tumor development. The detailed results of this study may have clinical implications regarding the decision of whether, and when, to use putative anti-Stat5 therapy [66].

\section{Abbreviations}

BLG: $\beta$-lactoglobulin; Stat5: signal transducer and activator of transcription 5; STAT5: transgenic Stat5; STAT5ca: constitutively active STAT5; STAT5 $\Delta 750$ : truncated STAT5; TAD: transactivation domain.

\section{Competing interests}

The authors declare that they have no competing interests. 


\section{Authors' contributions}

TE designed and performed the research and also helped in writing the manuscript. IB designed the research and wrote the manuscript.

\section{Additional material}

\section{Additional file 1}

Supporting information. Primers list and bioinformatical analyses of differentially expressed genes in tumors caused by the STAT5ca and STAT5 750 variants. Data sheet A. List of primers used to amplify coding regions of the following listed genes. Data sheet $B$. List of features with a significant $(\mathrm{P}<0.05)$, over twofold difference in expression between STAT5 750- and STAT5ca-induced tumors. Data sheet C. IPA classification of the differentially expressed genes in tumors induced by STAT5ca and the STAT5 750 transgenes. List of genes comprising the process annotation to biological function and/or disease is presented. Data sheet $D$.

Clustering of genes with a significant $(\mathrm{P}<0.05)$, over twofold difference in expression in tumors caused by the two STAT5 variants. Data sheet E. Clustering and IPA classification of the differentially expressed genes in tumors caused by STAT5ca and STAT5 750 variants. Data sheet F. List of specifically affected genes and those differentially expressed in each of the tumor sets relative to the mammary gland. Data sheet G. Set of 14 genes with expression levels that associate the effect of STAT5 750 with the resulting carcinoma phenotype and the effect of STAT5ca with the papillary adenocarcinoma phenotype.

Click here for file

[http://www.biomedcentral.com/content/supplementary/14712164-10-231-S1.xls]

\section{Additional file 2}

Correlations between the expression of STAT5ca and its specifically affected genes, and among genes specifically affected by the STAT5 variants. The table presents the correlation values between the expression levels of genes specifically affected by the STAT5ca and STAT5 750 variants in the tumors, and between the expression of STAT5ca and these genes.

Click here for file

[http://www.biomedcentral.com/content/supplementary/14712164-10-231-S2.doc]

\section{Acknowledgements}

This study was supported by a grant from the Israel Science Foundation, Israel Academy of Sciences, contract number: 706/04 to IB.

Contribution No. 536/08 from the ARO, The Volcani Center, Bet-Dagan, Israel.

\section{References}

I. Moriggl R, Gouilleux-Gruart V, Jahne R, Berchtold S, Gartmann C, Liu $X$, Hennighausen L, Sotiropoulos A, Groner B, Gouilleux F: Deletion of the carboxyl-terminal transactivation domain of MGFStat 5 results in sustained DNA binding and a dominant negative phenotype. Mol Cell Biol 1996, 16:5691-5700.

2. Bowman T, Garcia R, Turkson J, Jove R: STATs in oncogenesis. Oncogene 2000, 19:2474-2488.

3. Hendry L, John S: Regulation of STAT signalling by proteolytic processing. Eur J Biochem 2004, 27 I:46 I 3-4620.

4. Azam M, Erdjument-Bromage H, Kreider BL, Xia M, Quelle F, Basu R, Saris $C$, Tempst $P$, Ihle JN, Schindler C: Interleukin-3 signals through multiple isoforms of Stat5. Embo J 1995, I4: I 402- I4I I.
5. Meyer J, Jucker M, Ostertag W, Stocking C: Carboxyl-truncated STAT5beta is generated by a nucleus-associated serine protease in early hematopoietic progenitors. Blood 1998, 91:1901-1908.

6. Mitchell TJ, Whittaker SJ, John S: Dysregulated expression of COOH-terminally truncated Stat5 and loss of IL2-inducible Stat5-dependent gene expression in Sezary Syndrome. Cancer Res 2003, 63:9048-9054.

7. Xia Z, Sait SN, Baer MR, Barcos M, Donohue KA, Lawrence D, Ford LA, Block AM, Baumann H, Wetzler M: Truncated STAT proteins are prevalent at relapse of acute myeloid leukemia. Leuk Res 200I, 25:473-482.

8. Ilaria RL Jr, Van Etten RA: P2 10 and PI 90 (BCR/ABL) induce the tyrosine phosphorylation and DNA binding activity of multiple specific STAT family members. I Biol Chem 1996, 27I:31704-317I0.

9. Van Etten RA: Aberrant cytokine signaling in leukemia. Oncogene 2007, 26:6738-6749.

10. Wagner $\mathrm{KU}, \mathrm{Rui} \mathrm{H}$ : Jak2/Stat5 signaling in mammogenesis, breast cancer initiation and progression. J Mammary Gland Biol Neoplasia 2008, 13:93-103.

II. lavnilovitch E, Groner B, Barash I: Overexpression and forced activation of stat5 in mammary gland of transgenic mice promotes cellular proliferation, enhances differentiation, and delays postlactational apoptosis. Mol Cancer Res 2002, I:32-47.

12. lavnilovitch E, Eilon T, Groner B, Barash I: Expression of a carboxy terminally truncated Stat5 with no transactivation domain in the mammary glands of transgenic mice inhibits cell proliferation during pregnancy, delays onset of milk secretion, and induces apoptosis upon involution. Mol Reprod Dev 2006, 73:84I-849.

13. Cotarla I, Ren S, Zhang Y, Gehan E, Singh B, Furth PA: Stat5a is tyrosine phosphorylated and nuclear localized in a high proportion of human breast cancers. Int J Cancer 2004, 108:665-67।.

14. Humphreys RC, Hennighausen L: Signal transducer and activator of transcription $5 \mathrm{a}$ influences mammary epithelial cell survival and tumorigenesis. Cell Growth Differ 1999, 10:685-694.

15. lavnilovitch E, Cardiff RD, Groner B, Barash I: Deregulation of Stat5 expression and activation causes mammary tumors in transgenic mice. Int I Cancer 2004, I | 2:607-6 I9.

16. Eilon T, Groner B, Barash I: Tumors caused by overexpression and forced activation of Stat5 in mammary epithelial cells of transgenic mice are parity-dependent and developed in aged, postestropausal females. Int J Cancer 2007, I 21:1892-1902

17. Perou CM, Sorlie T, Eisen MB, Rijn M van de, Jeffrey SS, Rees CA, Pollack JR, Ross DT, Johnsen H, Akslen LA, Fluge O, Pergamenschikov A, Williams C, Zhu SX, Lønning PE, Børresen-Dale AL, Brown PO, Botstein D: Molecular portraits of human breast tumors. Nature 2000, 406:747-752.

18. van ' $t$ Veer LJ, Dai H, Vijver MJ van de, He YD, Hart AA, Mao M, Peterse HL, Kooy K van der, Marton MJ, Witteveen AT, Schreiber GJ, Kerkhoven RM, Roberts C, Linsley PS, Bernards R, Friend SH: Gene expression profiling predicts clinical outcome of breast cancer. Nature 2002, 4I5:530-536.

19. Ein-Dor L, Kela I, Getz G, Givol D, Domany E: Outcome signature genes in breast cancer: is there a unique set. Bioinformatics 2005, 21 : I7I-178.

20. Dressman HK, Hans C, Bild A, Olson JA, Rosen E, Marcom PK, Liotcheva VB, Jones EL, Vujaskovic Z, Marks J, Dewhirst MW, West $M$, Nevins JR, Blackwell K: Gene expression profiles of multiple breast cancer phenotypes and response to neoadjuvant chemotherapy. Clin Cancer Res 2006, I 2:819-826.

21. Ye Y, Qiu TH, Kavanaugh C, Green JE: Molecular mechanisms of breast cancer progression: lessons from mouse mammary cancer models and gene expression profiling. Breast Dis 2004, 19:69-82.

22. Qiu TH, Chandramouli GV, Hunter KW, Alkharouf NW, Green JE, Liu ET: Global expression profiling identifies signatures of tumor virulence in MMTV-PyMT-transgenic mice: correlation to human disease. Cancer Res 2004, 64:5973-598I.

23. Eilon T, Barash I: Different gene-expression profiles for the poorly differentiated carcinoma and the highly differentiated 
papillary adenocarcinoma in mammary glands support distinct metabolic pathways. BMC Cancer 2008, 8:270.

24. Desai KV, Xiao N, Wang W, Gangi L, Greene J, Powell Jl, Dickson R, Furth P, Hunter K, Kucherlapati R, Simon R, Liu ET, Green JE: Initiating oncogenic event determines gene-expression patterns of human breast cancer models. Proc Natl Acad Sci USA 2002, 99:6967-6972

25. Lauren PD: Algorithm to model gene expression on Affymetrix chips without the use of MM cells. IEEE Trans Nanobioscience 2003, 2:163-170.

26. Miller LD, Long PM, Wong L, Mukherjee S, McShane LM, Liu ET: Optimal gene expression analysis by microarrays. Cancer Cell 2002, 2:353-361.

27. Eisen MB, Spellman PT, Brown PO, Botstein D: Cluster analysis and display of genome-wide expression patterns. Proc Natl Acad Sci USA 1998, 95:|4863-|4868.

28. Yoshida $Y$, Togi K, Matsumae H, Nakashima $Y$, Kojima $Y$, Yamamoto $\mathrm{H}$, Ono K, Nakamura T, Kita T, Tanaka M: CCNI protects cardiac myocytes from oxidative stress via betal integrin-Akt pathway. Biochem Biophys Res Commun 2007, 355:6 I I-6I8.

29. Gressner OA, Lahme B, Siluschek M, Rehbein K, Herrmann J, Weiskirchen R, Gressner AM: Activation of TGF-beta within cultured hepatocytes and in liver injury leads to intracrine signaling with expression of connective tissue growth factor. J Cell Mol Med 2008, I 2:27। 7-2730.

30. Yu Y, Gao Y, Wang H, Huang L, Qin J, Guo R, Song M, Yu S, Chen J, Cui B, Gao P: The matrix protein CCNI (CYR6I) promotes proliferation, migration and tube formation of endothelial progenitor cells. Exp Cell Res 2008, 3 I 4:3198-3208.

31. O'Kelly J, Chung A, Lemp N, Chumakova K, Yin D, Wang HJ, Said J, Gui D, Miller CW, Karlan BY, Koeffler HP: Functional domains of CCNI (Cyr6I) regulate breast cancer progression. Int I Oncol 2008, 33:59-67.

32. Egea M, Meton I, Cordoba M, Fernandez F, Baanante IV: Role of Sp I and SREBP-Ia in the insulin-mediated regulation of glucokinase transcription in the liver of gilthead sea bream (Sparus aurata). Gen Comp Endocrinol 2008, 155:359-367.

33. Fukumoto S, Miner JH, Ida H, Fukumoto E, Yuasa K, Miyazaki H, Hoffman MP, Yamada Y: Laminin alpha5 is required for dental epithelium growth and polarity and the development of tooth bud and shape. J Biol Chem 2006, 281:5008-5016.

34. Ikeda W, Nakanishi H, Takekuni K, Itoh S, Takai Y: Identification of splicing variants of Frabin with partly different functions and tissue distribution. Biochem Biophys Res Commun 200I, 286: 1066-1072.

35. Ruckhaberle E, Rody A, Engels K, Gaetje R, von Minckwitz G, Schiffmann S, Grosch S, Geisslinger G, Holtrich U, Karn T, Kaufmann M: Microarray analysis of altered sphingolipid metabolism reveals prognostic significance of sphingosine kinase $I$ in breast cancer. Breast Cancer Res Treat 2008, I I 2:41-52.

36. Catalan V, Gomez-Ambrosi J, Pastor C, Rotellar F, Silva C, Rodriguez A, Gil MJ, Cienfuegos JA, Salvador J, Vendrell J, Frühbeck G: Influence of Morbid Obesity and Insulin Resistance on Gene Expression Levels of AQP7 in Visceral Adipose Tissue and AQP9 in Liver. Obes Surg 2008, 18:695-70।.

37. Shin JH, Choi GS, Kang WH, Myung KB: Sphingosine I-phosphate triggers apoptotic signal for $B 16$ melanoma cells via ERK and caspase activation. J Korean Med Sci 2007, 22:298-304.

38. Kim IJ, Lee KW, Park BY, Lee JK, Park J, Choi IY, Eom S!, Chang TS, Kim MJ, Yeom YI, Chang SK, Lee YD, Choi EJ, Han PL: Molecular cloning of multiple splicing variants of JIP-I preferentially expressed in brain. I Neurochem 1999, 72:1335-1343.

39. Uehara N, Unami A, Kiyozuka Y, Shikata N, Oishi Y, Tsubura A: Parous mammary glands exhibit distinct alterations in gene expression and proliferation responsiveness to carcinogenic stimuli in Lewis rats. Oncol Rep 2006, 15:903-91।

40. Emslie D, D'Costa K, Hasbold J, Metcalf D, Takatsu K, Hodgkin PO, Corcoran LM: Oct2 enhances antibody-secreting cell differentiation through regulation of IL-5 receptor alpha chain expression on activated B cells. J Exp Med 2008, 205:409-42 I.

41. Jimenez MA, Akerblad P, Sigvardsson M, Rosen ED: Critical role for Ebfl and Ebf 2 in the adipogenic transcriptional cascade. Mol Cell Biol 2007, 27:743-757.

42. Mahajan KN, Mitchell BS: Role of human Pso4 in mammalian DNA repair and association with terminal deoxynucleotidyl transferase. Proc Natl Acad Sci USA 2003, 100:10746-1075I.
43. Hong J, Doebele RC, Lingen MW, Quilliam LA, Tang WJ, Rosner MR: Anthrax edema toxin inhibits endothelial cell chemotaxis via Epac and Rap I. J Biol Chem 2007, 282: I978I-I 9787.

44. Wang-Rodriguez J, Urquidi V, Rivard A, Goodison S: Elevated osteopontin and thrombospondin expression identifies malignant human breast carcinoma but is not indicative of metastatic status. Breast Cancer Res 2003, 5:R /36-143.

45. Dennis G Jr, Sherman BT, Hosack DA, Yang J, Gao W, Lane HC, Lempicki RA: DAVID: Database for Annotation, Visualization, and Integrated Discovery. Genome Biol 2003, 4:P3

46. Cui Y, Riedlinger G, Miyoshi K, Tang W, Li C, Deng CX, Robinson GW, Hennighausen L: Inactivation of Stat5 in mouse mammary epithelium during pregnancy reveals distinct functions in cell proliferation, survival, and differentiation. Mol Cell Biol 2004, 24:8037-8047.

47. Pfitzner E, Jahne R, Wissler M, Stoecklin E, Groner B: p300/CREBbinding protein enhances the prolactin-mediated transcriptional induction through direct interaction with the transactivation domain of Stat5, but does not participate in the Stat5-mediated suppression of the glucocorticoid response. Mol Endocrinol 1998, I 2:1582-1593.

48. Rascle A, Johnston JA, Amati B: Deacetylase activity is required for recruitment of the basal transcription machinery and transactivation by STAT5. Mol Cell Biol 2003, 23:4I62-4I73.

49. Rascle A, Lees E: Chromatin acetylation and remodeling at the Cis promoter during STAT5-induced transcription. Nucleic Acids Res 2003, 31 : 6882-6890.

50. Weidner KM, Sachs M, Birchmeier W: The Met receptor tyrosine kinase transduces motility, proliferation, and morphogenic signals of scatter factor/hepatocyte growth factor in epithelial cells. I Cell Biol 1993, I 2 I: | 45- I54.

5I. Lindemann K, Resau J, Nahrig J, Kort E, Leeser B, Annecke K, Welk A, Schafer J, Woude GF Vande, Lengyel E, Harbeck N: Differential expression of c-Met, its ligand HGF/SF and HER2/neu in DCIS and adjacent normal breast tissue. Histopathology 2007, $51: 54-62$.

52. Ghoussoub RA, Dillon DA, D'Aquila T, Rimm EB, Fearon ER, Rimm $\mathrm{DL}$ : Expression of c-met is a strong independent prognostic factor in breast carcinoma. Cancer 1998, 82:1513-1520.

53. Wang XY, Yin Y, Yuan H, Sakamaki T, Okano H, Glazer RI: Musashi I modulates mammary progenitor cell expansion through proliferin-mediated activation of the Wnt and Notch pathways. Mol Cell Biol 2008, 28:3589-3599.

54. Singh SK, Moretta D, Almaguel F, Wall NR, De Leon M, De Leon D: Differential effect of prolGF-II and IGF-II on resveratrol induced cell death by regulating survivin cellular localization and mitochondrial depolarization in breast cancer cells. Growth Factors 2007, 25:363-372.

55. Cardiff RD, Wellings SR: The comparative pathology of human and mouse mammary glands. J Mammary Gland Biol Neoplasia 1999, 4:105-122.

56. Gallego MI, Bierie B, Hennighausen L: Targeted expression of HGF/SF in mouse mammary epithelium leads to metastatic adenosquamous carcinomas through the activation of multiple signal transduction pathways. Oncogene 2003, 22:8498-8508.

57. Welm AL, Kim S, Welm BE, Bishop JM: MET and MYC cooperate in mammary tumorigenesis. Proc Natl Acad Sci USA 2005, 102:4324-4329.

58. Woelfle U, Cloos J, Sauter G, Riethdorf L, Janicke F, van Diest P, Brakenhoff R, Pantel K: Molecular signature associated with bone marrow micrometastasis in human breast cancer. Cancer Res 2003, 63:5679-5684.

59. Varambally S, Dhanasekaran SM, Zhou M, Barrette TR, Kumar-Sinha C, Sanda MG, Ghosh D, Pienta KJ, Sewalt RG, Otte AP, Rubin MA, Chinnaiyan AM: The polycomb group protein EZH2 is involved in progression of prostate cancer. Nature 2002, 419:624-629.

60. Chang Y, Zuka M, Perez-Pinera P, Astudillo A, Mortimer J, Berenson JR, Deuel TF: Secretion of pleiotrophin stimulates breast cancer progression through remodeling of the tumor microenvironment. Proc Natl Acad Sci USA 2007, 104: 10888- 10893.

61. Rousseau B, Larrieu-Lahargue F, Javerzat S, Guilhem-Ducleon F, Beermann F, Bikfalvi A: The tyrpI-Tag/tyrpI-FGFRI-DN bigenic mouse: a model for selective inhibition of tumor development, angiogenesis, and invasion into the neural tissue by 
blockade of fibroblast growth factor receptor activity. Cancer Res 2004, 64:2490-2495.

62. Yeh WC, Itie A, Elia AJ, Ng M, Shu HB, Wakeham A, Mirtsos C, Suzuki N, Bonnard M, Goeddel DV, Mak TW: Requirement for Casper (c-FLIP) in regulation of death receptor-induced apoptosis and embryonic development. Immunity 2000, I 2:633-642.

63. Zhao LY, Niu Y, Santiago A, Liu J, Albert SH, Robertson KD, Liao D: An EBF3-mediated transcriptional program that induces cell cycle arrest and apoptosis. Cancer Res 2006, 66:9445-9452.

64. Marenduzzo D, Faro-Trindade I, Cook PR: What are the molecular ties that maintain genomic loops? Trends Genet 2007, 23:126-133.

65. Trinkle-Mulcahy L, Lamond Al: Nuclear functions in space and time: gene expression in a dynamic, constrained environment. FEBS Lett 2008, 582:1960-1970.

66. Desrivieres S, Kunz C, Barash I, Vafaizadeh V, Borghouts C, Groner $B$ : The biological functions of the versatile transcription factors STAT3 and STAT5 and new strategies for their targeted inhibition. J Mammary Gland Biol Neoplasia 2006, I I:75-87.

Publish with Bio Med Central and every scientist can read your work free of charge

"BioMed Central will be the most significant development for disseminating the results of biomedical research in our lifetime. "

Sir Paul Nurse, Cancer Research UK

Your research papers will be:

- available free of charge to the entire biomedical community

- peer reviewed and published immediately upon acceptance

- cited in PubMed and archived on PubMed Central

- yours - you keep the copyright

Submit your manuscript here:

http://www.biomedcentral.com/info/publishing_adv.asp
BioMedcentral 